\title{
First CoRoT light curves of RR Lyrae stars
}

\section{Complex multiplet structure and non-radial pulsation detections in V1127 Aquilae $\star, \star \star$}

\author{
M. Chadid ${ }^{1}$, J. M. Benkő ${ }^{2}$, R. Szabó ${ }^{2}$, M. Paparó ${ }^{2}$, E. Chapellier ${ }^{1}$, K. Kolenberg ${ }^{3}$, E. Poretti ${ }^{4}$, \\ G. Bono ${ }^{5,6}$, J.-F. Le Borgne ${ }^{7}$, H. Trinquet ${ }^{1}$, S. Artemenko ${ }^{8}$, M. Auvergne ${ }^{9}$, A. Baglin ${ }^{9}$, J. Debosscher ${ }^{10}$, \\ K. N. Grankin ${ }^{8}$, E. Guggenberger ${ }^{3}$, and W. W. Weiss ${ }^{3}$
}

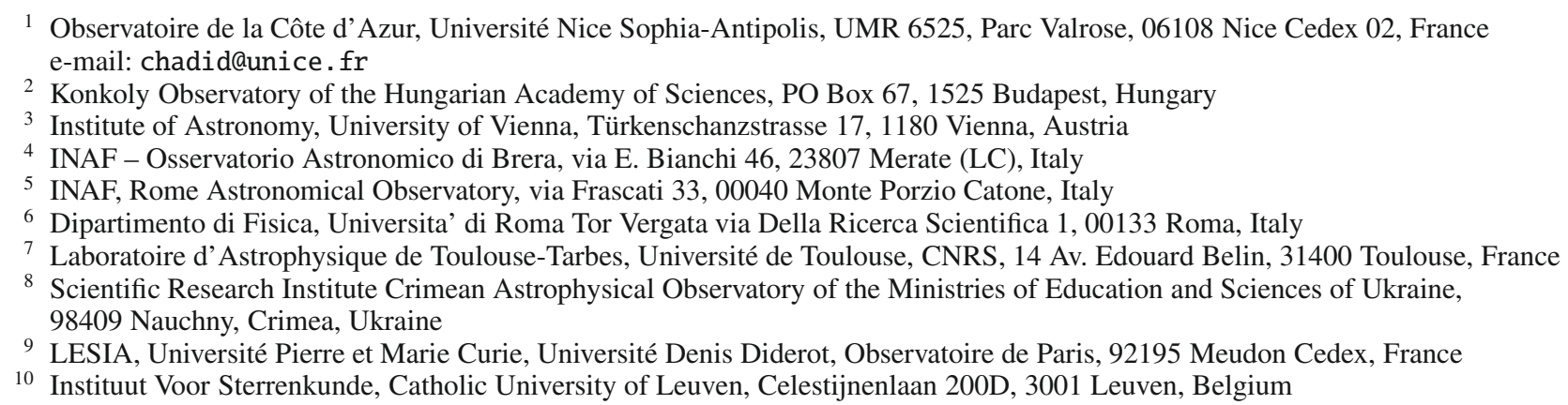

Received 29 September 2009 / Accepted 17 November 2009

\begin{abstract}
Context. The CoRoT - Convection Rotation and planetary Transits - space mission is a great opportunity for monitoring stars with excellent time-sampling and unprecedented photometric precision for up to 150 days. As an important benefit, high-quality RR Lyrae light curves are obtained with a quasi-uninterrupted coverage over several pulsation and Blazhko cycles.

Aims. The Blazhko effect in RR Lyrae stars is an unsolved problem of astrophysics. We used the high-precision space data to contribute more precise knowledge to explain the possible physical processes behind the phenomenon.

Methods. We applied different period-finding techniques including Period04, MuFrAn, PDM and SigSpec. Amplitude and phase modulation were investigated by an analytical function method as well as with the traditional $\mathrm{O}-\mathrm{C}$ diagrams.

Results. The Blazhko modulation frequency is directly detected in the spectrum, as well as its first and second harmonics. It shows the non-linear nature of the Blazhko modulation. Besides the triplets, further higher-order modulation side peaks appear around the pulsation frequency as quintuplet, septuplet, nonuplet, undecaplet, tredecaplet, quindecaplet and sepdecaplet structures. Additional frequencies, not belonging to the classical multiplet structures, are detected, as well as their linear combinations with the fundamental radial mode. We interpret these additional terms as non-radial modes.

During the five consecutive Blazhko cycles, there is a shift of the maximum phase around 0.011 pulsation phase which is likely the consequence of a long term modulation.
\end{abstract}

Key words. stars: atmospheres - stars: variables: RR Lyr - techniques: photometric - methods: data analysis - hydrodynamics space vehicles

\section{Introduction}

RR Lyrae stars have contributed to almost every branch of modern astronomy. They are the most important low-mass distance indicators (Del Principe et al. 2006; Sollima et al. 2008), they are present in all the stellar systems including a stellar population older than a few Gyrs (Sanna et al. 2009). Moreover, they are fundamental benchmarks to constrain the plausibility

* Full Table 1 is only available in electronic form at the CDS via anonymous ftp to cdsarc.u-strasbg.fr (130.79.128.5) or via http://cdsweb.u-strasbg. fr/cgi-bin/qcat?J/A+A/510/A39

$\star \star$ The CoRoT space mission was developed and is operated by the French space agency CNES, with participation of ESA's RSSD and Science Programmes, Austria, Belgium, Brazil, Germany, and Spain. of the physical assumptions adopted to construct both evolutionary and pulsation models of low-mass stars (Del Principe et al. 2005). In recent years, their atmosphere has been considered as a laboratory of hypersonic shock wave simulation (Chadid et al. 2008). With their large amplitudes, the RR Lyrae stars have been known for more than a century. Although these stars are well studied, a few disturbing questions concerning their pulsation and their atmospheric dynamics remain to be answered. In particular the Blazhko modulation is known since about hundred years, while its physical origin remains an enigma. The Blazhko effect (Blazhko 1907) is a periodic modulation of both the amplitude and the phase of the main pulsation on timescales typically varying from dozens to hundreds of days. As discussed by Chadid \& Chapellier (2006) and Chadid et al. (2004), none 
of the currently suggested models - the resonance model which involves the non-radial modes (Dziembowski \& Mizerski 2004) and the magnetic oblique rotator (Shibahashi 2000) - is able to explain the complexity of the observed properties of the Blazhko modulation. Recently, Stothers (2006) gave a new interpretation of the modulating Blazhko effect in RR Lyrae stars. He explains the continuous amplitude and period changes of the main pulsation by a convective turbulent dynamo inside the hydrogen and helium ionization zones, where the dynamo period is identical to the Blazhko period. The Stothers theory does not require any non-radial mode, but it still remains a qualitative interpretation of the modulation which needs to be confirmed.

Many studies have been devoted to the light curve changes of RR Lyrae stars. Initially, the observations were biased in general towards the rising branch and maximum phase of the light curve (Walraven 1949). Even the best of them have some defects caused by the inaccuracy and data inhomogeneity. On the other hand, both photometric (e.g. Borkowski 1980; Kovács 1995; Smith et al. 2003) and spectroscopic observations (Struve \& Blaauw 1949; Preston et al. 1965; Chadid et al. 1999; Chadid \& Chapellier 2006) of well-studied field Blazhko stars, have yielded accurate frequency spectra which allow the study of the pulsation characteristics in detail. However, the large gaps typical of inadequately sampled earth-bound single-site observations sometimes hinder an unambiguous determination of the frequencies and their amplitudes and phases. Even if the outcome of large-scale studies such as microlensing surveys (OGLE, see Moskalik \& Poretti 2003 and MACHO, see Alcock et al. 2000, 2003) has yielded a much better picture of the general phenomenology of the Blazhko effect by the extensive multi-target surveys, these are not extended enough to study the modulation properties in full detail. Multisite campaigns (Kolenberg et al. 2006) and compact dedicated single-site multicolour observations (Jurcsik et al. 2005, 2006, 2008, 2009) provided a higherlevel knowledge on the Blazhko phenomenon, but the coverage is still not continuous on a long time base.

The CoRoT space mission (Convection Rotation and Planetary Transits), successfully launched on 2006 December 27, is a great opportunity for monitoring the pulsating stars with excellent time-sampling and unprecedented photometric precision for up to 150 days. As an important consequence, several high-quality RR Lyrae light curves are obtained with a quasi-uninterrupted coverage over several pulsation and Blazhko cycles with an excellent photometric accuracy. Our team, the RR Lyrae-CoRoTeam ${ }^{1}$ is an international collaboration focusing on a better understanding of RR Lyrae stars using CoRoT data. Our first RR Lyrae CoRoT study has been focused on RR Lyrae stars found during the first long run of the CoRoT mission, long run centre and anticentre - LRc01 and LRa01 - of the Milky Way. We classified six RR Lyrae stars in LRc01, four of them are RRab Blazhko stars (CoRoT ID: 0100689962, 0101128793, 0101503544 and 0100881648) one of them is an RRab monoperiodic (CoRoT ID:0101370131) star and one is an RRd star (CoRoT ID:0101368812) and in LRa01, there is also one RR ab monoperiodic star (CoRoT ID:0102781750) (see Chadid et al. 2009).

In this paper we present the results of the photometric CoRoT data on the star with CoRoT ID: 0100689962, known as V1127 Aql ( $\alpha=19^{\mathrm{h}} 24^{\mathrm{m}} 00^{\mathrm{s}} .11, \delta=+01^{\circ} 41^{\prime} 48^{\prime \prime} .9$, J2000).

The variability of the star was discovered by Hoffmeister (1966) on the Sonneberg plates. Since then, observations

1 The dedicated website is http://fizeau. unice.fr/corot devoted to V1127 Aql have not been published apart from some early photographic maxima measurements (Gessner 1973).

The CoRoT data of V1127 Aql were obtained continuously and regularly from 16 May 2007 to 05 October 2007. Note that the CoRoTJD corresponds to HJD 2451545.0 (January 1st, 2000 at $\left.12^{\mathrm{h}}: 00^{\mathrm{m}}: 00^{\mathrm{s}}\right)$. In Sect. 2. we present CoRoT observations. Section 3. is devoted to the description of data analysis and its results with new findings. The specialities of the Blazhko phenomenon are discussed in Sect. 4. Finally some concluding remarks are given in Sect. 5.

\section{Observations and data processing}

The CoRoT data used in this study are the calibrated light curves, N2-level, that have been measured by the CoRoT Exoplanet CCDs during the first long run in the centre direction of the Galaxy. The total time span is 143 days. The time sampling of the light curves is $32 \mathrm{~s}$, an average is taken on board over 16 such measurements, and these results in a nominal sampling time of $512 \mathrm{~s}$. We used here the resulting "white" flux for our purpose, and we removed all measurements having non-zero quality flags in the N2 product delivery, retaining only valid flux measurements. These flagged measurements included the measurements which were taken during the South-Atlantic Anomaly passages. The bad data points account for $2 \%$ of the data and, their removal breaks the equidistancy of the time series and changes the behaviour of the window function. For more technical details on the satellite and data we refer to Auvergne et al. (2009). The CoRoT N2 data are affected by instrumental trends and jumps in the light curves due to changes in the amount of incident stray light during the run, periodic changes in flux caused by the satellite orbit, and discontinuities in the light curves due to cosmic ray hits on the CCDs. Then, after the removal of bad data points a trend and jump filtering code was used to the data. The trend filtering part of the process divides the data set into bins and within each bin the fluxes and times are averaged. The independently binned, averaged values of fluxes are interpolated in the original points of time and subtracted from the observed ones. The jump filtering process is based on the difference of the subsequent points of the light curve. A difference is regarded as a jump if it exceeds a certain factor of the standard deviation. The data are transformed into a zero averaged instrumental magnitude scale.

\section{Frequency analysis}

As is well known, the Fourier spectra of classical Blazhko RR Lyrae stars are dominated by equidistant triplets with a frequency separation identical with the modulation frequency (Chadid et al. 1999; Chadid \& Chapellier 2006; Kolenberg et al. 2006). Recently, Hurta et al. (2008) found equidistant quintuplets in the spectrum of RV UMa. The appearance of the Blazhko modulation frequency $f_{\mathrm{m}}$ itself was a long-standing debate. However, it has always been detected in the spectra of extended enough photometric data sets (Kovács 1995; Nagy 1998; Jurcsik et al. 2005, 2006, 2008, 2009). In the case of MW Lyr it was found together with its first harmonics $2 f_{\mathrm{m}}$ (Jurcsik et al. 2008).

From a purely mathematical point of view, when an arbitrary periodic signal is modulated both in its amplitude and phase, its Fourier spectrum shows an infinite series of side peak structures with asymmetric amplitudes between the higher and lower frequency sides (Benkố et al. 2009; Szeidl \& Jurcsik 2009). 


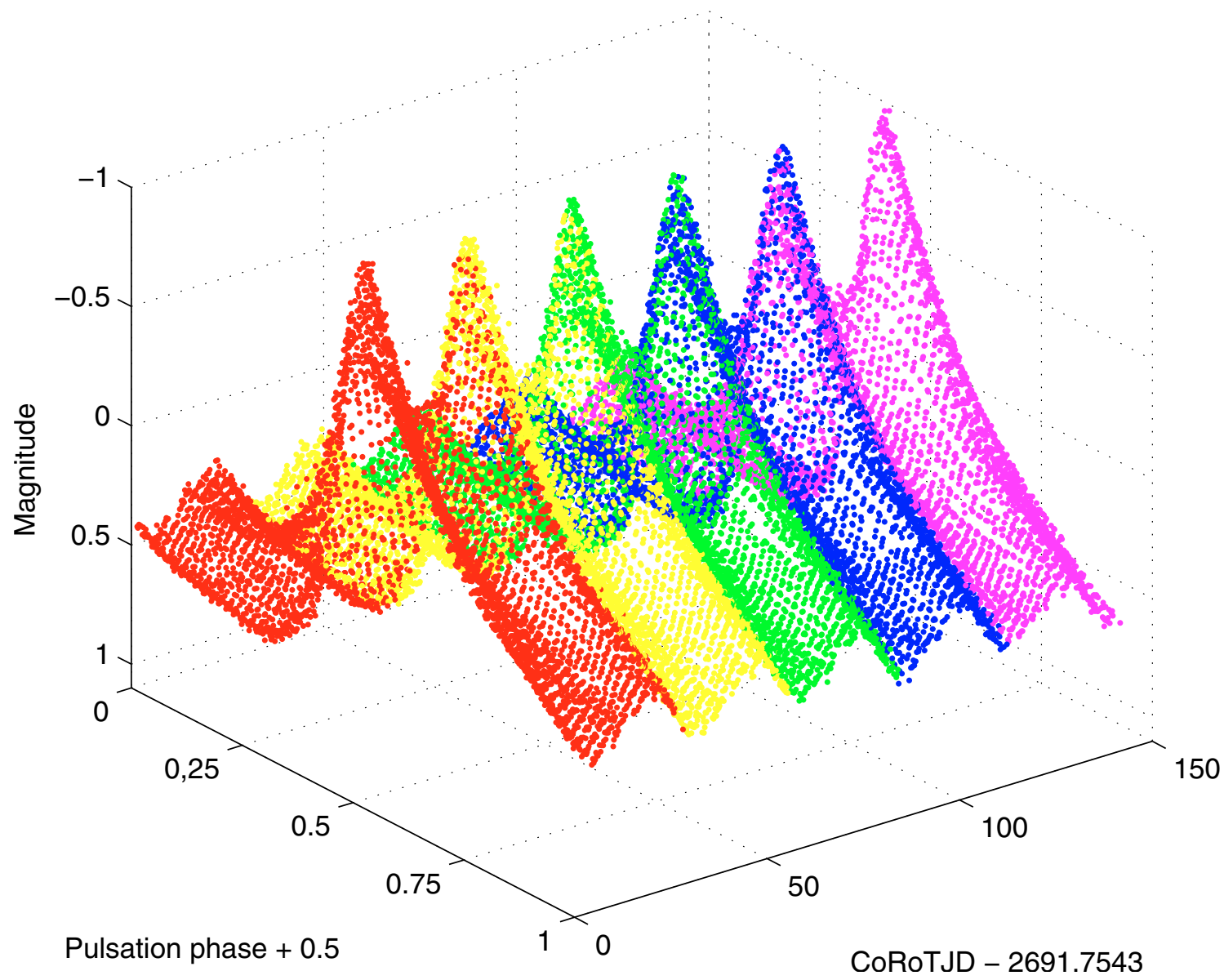

Fig. 1. Two-dimensional CoRoT light curve of V1127 Aql folded with the pulsation period (0.355996 d) over five Blazhko cycles. The variations in the light curve due to the Blazhko effect are clearly visible.

\subsection{Detection of frequencies}

The CoRoT data of V1127 Aql provide 400 consecutive pulsation cycles and a homogeneous coverage of five Blazhko cycles. The heights of maxima and minima of the light curves clearly indicate a modulation period of about 27 days (Fig. 1).

We used different algorithms to find frequencies, such as Period04 (Lenz \& Breger 2005), the package MuFrAn (Kolláth 1990), the CLEAN algorithm as introduced by Roberts et al. (1987) and the PDM method (Stellingwerf 1978). The frequency analyses all led to the same results with a slight difference at higher orders. In this study, we present the results of the MuFrAn frequency analysis. The Fourier decomposition consists of fitting the magnitude measurements by means of the series

$m(t)=A_{0}+\sum_{i=1, N} A_{i} \sin \left[2 \pi F_{i}\left(t-T_{0}\right)+\Phi_{i}\right]$,

where $T_{0}$ is the initial epoch value of the data set CoRoTJD $T_{0}=$ 2691.0.

The original spectrum is dominated by the main pulsation frequency $F_{1}=f_{0}=2.809017 \mathrm{~d}^{-1}$, its harmonics up to the 20th order (Fig. 2a) and the modulation triplet structure $n f_{0} \pm f_{\mathrm{m}}$ (as insert panel a). After prewhitening the spectrum with the main pulsation frequency, its harmonics and the triplet structure, the dominant peaks are at the frequencies of higher-order side peaks $n f_{0} \pm k f_{\mathrm{m}}, k \neq 1$ (Fig. 2b) and at the modulation frequency $f_{\mathrm{m}}=0.0372 \mathrm{~d}^{-1}$. The unexpected amplitude ratio of the multiplets will be discussed later. The insert in panel $b$ shows the higher-order side peak structure. Figure 3 shows a map of harmonics and side peak structures, listed in Tables 1.A.1, 1.A.3, 1.A.4, 1.A.5, 1.A.6, 1.A.7, 1.A.8, 1.A.9, and 1.A.10. We plotted the order of side peaks $k$ versus harmonic orders $n$ in the formula $n f_{0}+k f_{\mathrm{m}}$. Due to the high precision of the CoRoT data the number of observed side peaks remarkably increased compared to numbers known up to now. Altogether 161 side peaks were identified in the frequency spectrum. Surprisingly, the lower harmonics have fewer side peaks. At the same time we could identify the multiplet structure around the higher harmonics where the harmonic itself was not significantly detected.

Figure $2 \mathrm{~b}$ shows the presence of Blazhko modulation frequency as well. We clearly detected not only the modulation frequency $f_{\mathrm{m}}=0.0372 \mathrm{~d}^{-1}$ but also its first $2 f_{\mathrm{m}}=0.0744$ and second harmonics $3 f_{\mathrm{m}}=0.1116$ in V1127 Aql (Fig. 4; Table 1.A.2). Figure $2 \mathrm{c}$ shows the spectrum after the prewhitening with modulation frequency, its harmonics and all higher-order side peaks. The dominant peak $f^{\prime}=4.0326 \mathrm{~d}^{-1}$ in the spectrum does not fit to the regular pattern of harmonics and side peaks in an RR Lyrae star.

Figure $2 \mathrm{~d}$ shows the residual spectrum prewhitening of all the frequencies we identified. To check our frequency solution we ran the program package SigSpec. It calculates the spectral significance defined by Reegen (2007) for each peak of the spectrum. The critical point of where to stop in the frequency analyses is a well-known, unsolved problem. Generally, the application of a certain signal-to-noise ratio is used to be accepted as a significance level. In our case sig $=5.2$ value served as an overall criterium. However, the regular behaviour of harmonics and side peaks in the Blazhko RR Lyrae star helps to identify even 


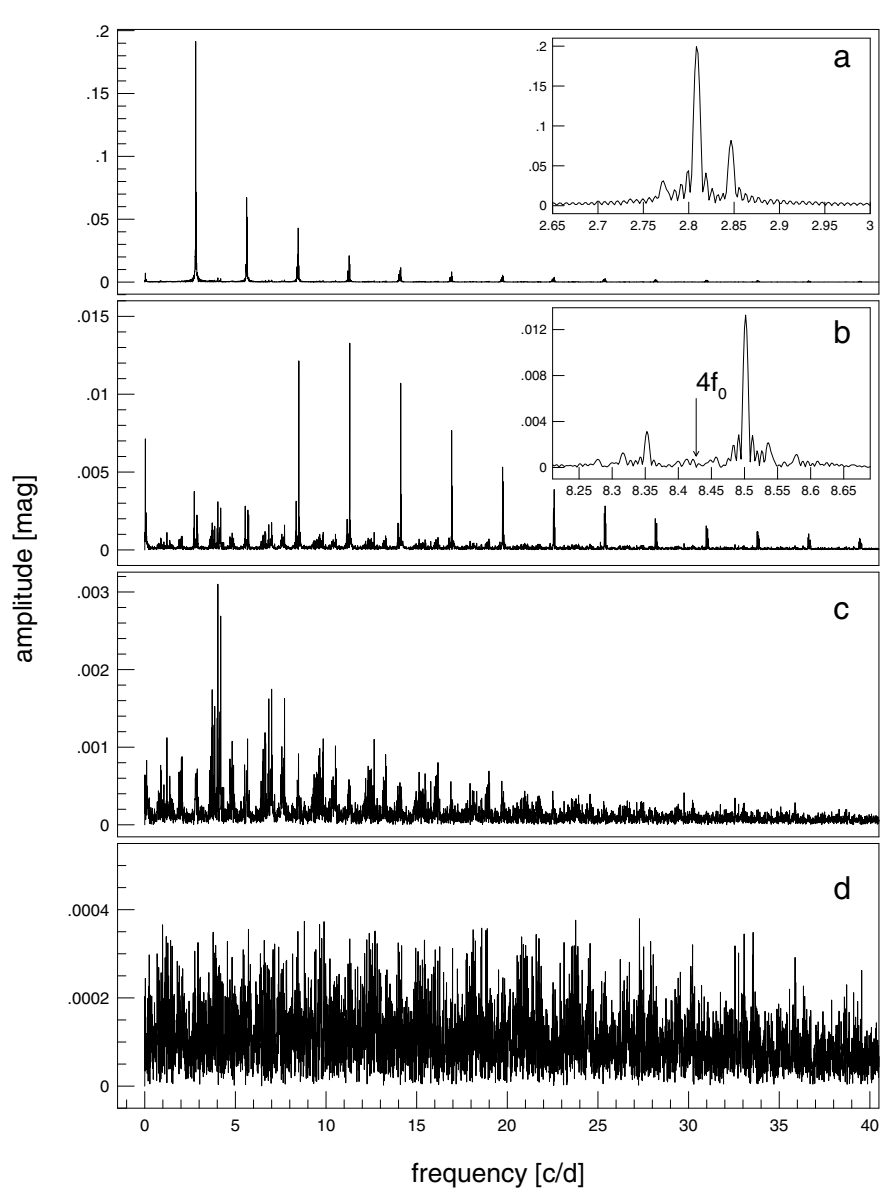

Fig. 2. The main steps of prewhitening process. a) The amplitude spectrum. b) Prewhitened spectrum with main pulsation frequency, its harmonics and the triplets (see insert in panel a)). c) Prewhitened spectrum with modulation frequency, its harmonics and all higher-order side peaks (see insert in panel b)). d)) Residual spectrum when all frequencies in Table 1 are removed. (For better visibility only the lower frequency regions are shown.)

frequency peaks, intrinsic to the star's pulsation, with a lower amplitude than the accepted value. Through a rigorous check in the high-frequency region, where the noise level is lower, we were able to detect the harmonics and their side peaks below the generally accepted significance level. An example is presented in Fig. 5. Around the 17th harmonic among the marked peaks only the five highest amplitude peaks have a higher value than the accepted significance level. Nevertheless, the regularity of the Blazhko side peak structure can be nicely followed among the lower amplitude peaks. We included these frequencies in our solution. Comparing our frequencies to the result of the SigSpec we conclude that they are very similar if we set the parameter $\mathrm{Sig} \approx 5$. We add the spectral significances from SigSpec to the amplitudes and phases of the finally accepted frequencies (Table 1).

Knowing the precise values of the pulsation and Blazhko period we deduce the 2007 Blazhko ephemeris:

$\operatorname{CoRoTJD}(\max )=2695.2028+(26.88 \pm 0.05) \mathrm{E}$,

and the 2007 pulsation ephemeris:

$\operatorname{CoRoTJD}(\max )=2695.2028+(0.3559966 \pm 0.0000006) \mathrm{E}$.

In this paper, the pulsation phase is $\phi$ and the Blazhko phase is $\psi$.
Table 1. Frequencies, Fourier amplitudes, phases, spectral significances and identification of the frequencies (this table can be found in its entirety at CDS).

\begin{tabular}{rcrrl}
\hline \hline $\begin{array}{c}\text { Frequency } \\
{\left[\mathrm{d}^{-1}\right]}\end{array}$ & $\begin{array}{c}\text { Amplitude } \\
{[\mathrm{mag}]}\end{array}$ & $\begin{array}{c}\text { Phase } \\
{[\mathrm{deg}]}\end{array}$ & Sig & ID \\
\hline 2.809017 & 0.20132 & 317.51 & 2912.65 & $f_{0}$ \\
5.618034 & 0.08213 & 50.81 & 1391.78 & $2 f_{0}$ \\
8.427051 & 0.03661 & 142.77 & 985.08 & $3 f_{0}$ \\
11.236067 & 0.01391 & 244.49 & 537.77 & $4 f_{0}$ \\
14.045084 & 0.00600 & 329.51 & 193.43 & $5 f_{0}$ \\
16.854101 & 0.00218 & 23.01 & 38.98 & $6 f_{0}$ \\
19.663118 & 0.00205 & 71.91 & 52.56 & $7 f_{0}$ \\
22.472135 & 0.00208 & 165.19 & 56.28 & $8 f_{0}$ \\
25.281152 & 0.00165 & 262.38 & 41.69 & $9 f_{0}$ \\
28.090169 & 0.00109 & 355.08 & 26.97 & $10 f_{0}$ \\
$\ldots$ & $\ldots$ & $\ldots$ & $\cdots$ & $\cdots$ \\
\hline
\end{tabular}

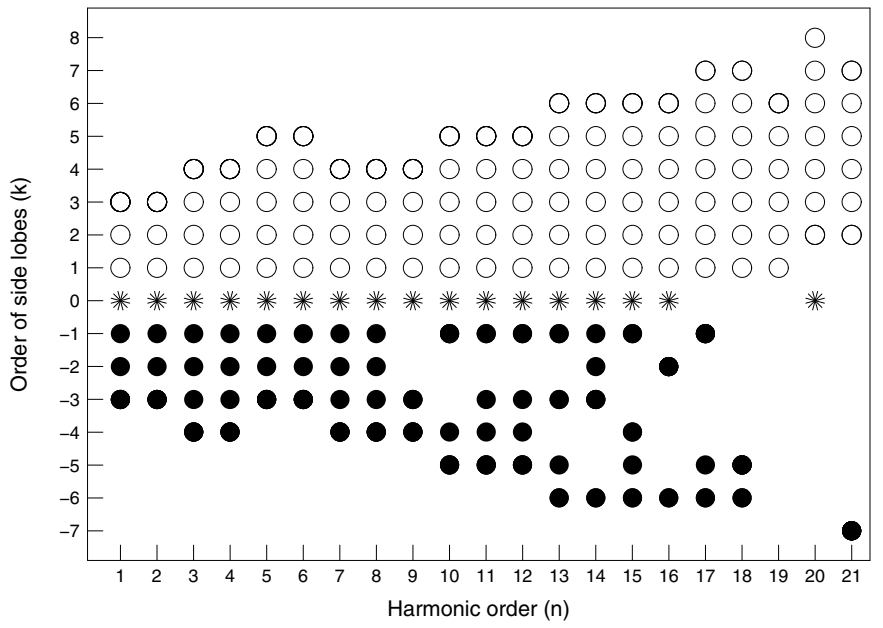

Fig. 3. Map of harmonics and side peaks in the Fourier spectrum of the V1127 Aql. The order of side frequencies $(k)$ vs. harmonic orders $(n)$ is plotted according to the formula $n f_{0}+k f_{\mathrm{m}}$. Open circles denote $k>0$, filled circles $k<0$ and stars $k=0$, respectively.

\subsection{Blazhko multiplets}

The complex side peak structure shown in Fig. 3 is highly connected to the amplitude value of certain peaks both in the case of harmonics and Blazhko side peaks. The amplitude of the harmonics of the main frequency seemingly decreases exponentially as it is obtained in RR Lyrae stars. However, presenting them on a logarithmic scale a standstill appears at the 6th, 7th and 8th harmonics (Fig. 6). The amplitude of these harmonics are the same while the lower-order harmonics and the higherorder harmonics are situated on straight lines with a slightly different inclination. An abrupt increase of the higher-order harmonics' amplitudes was reported only on the other CoRoT RR Lyrae star ID:0101370131 (Paparó et al. 2009).

Blazhko side peaks with non-significant amplitudes are omitted causing holes in the regular pattern in Fig. 3. Table 2 summarizes the type of multiplet structure of V1127 Aql. The triplet, quintuplet and septuplet structures are more numerous in agreement with the fact that these are more frequently obtained in lower quality data sets. For nonuplet (9 peaks) to septdecaplet (17 peaks) structures the number of findings is decreasing due to the lower amplitudes. 


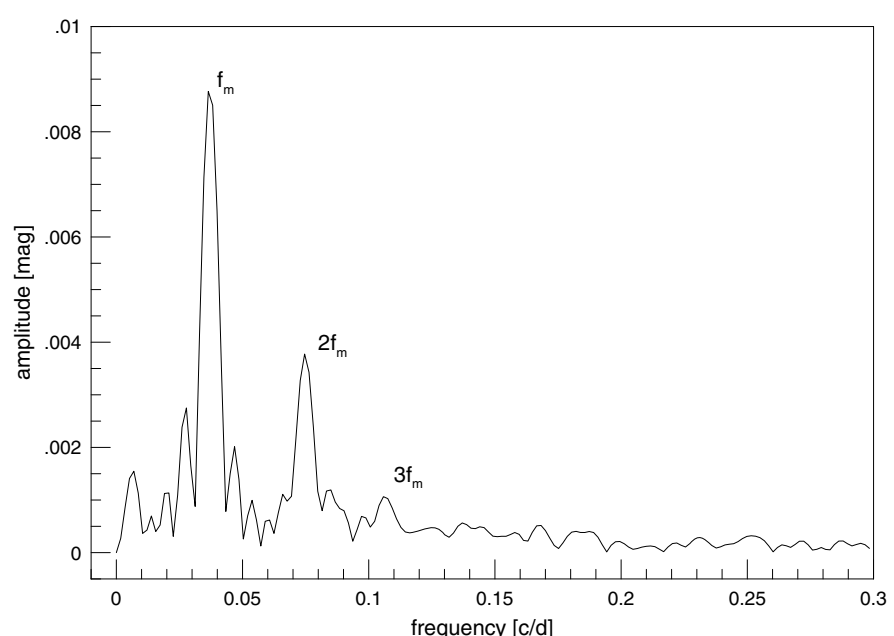

Fig. 4. The Blazhko modulation frequency $f_{\mathrm{m}}=0.0372 \mathrm{~d}^{-1}$ and its harmonics $2 f_{\mathrm{m}}, 3 f_{\mathrm{m}}$ are clearly visible in the spectrum of V1127 Aql.

Table 2. Complex multiplet structures, degree of deviation and degree of asymmetry.

\begin{tabular}{lccl}
\hline \hline Multiplet & No. of freq. & $\begin{array}{c}\delta f \\
{\left[\mathrm{~d}^{-1}\right]}\end{array}$ & $Q-\bar{Q}$ \\
\hline Triplet & 34 & 0.000093 & 0.43 \\
Quintuplet & 31 & -0.000115 & -0.1 \\
Septuplet & 34 & 0.003519 & -0.21 \\
Nonuplet & 28 & 0.001067 & 0.21 \\
Undecaplet & 21 & 0.0026 & 0.18 \\
Tredecaplet & 15 & 0.0045 & -0.77 \\
Quindecaplet & 5 & 0.008276 & 0.35 \\
Septdecaplet & 1 & & \\
\hline
\end{tabular}

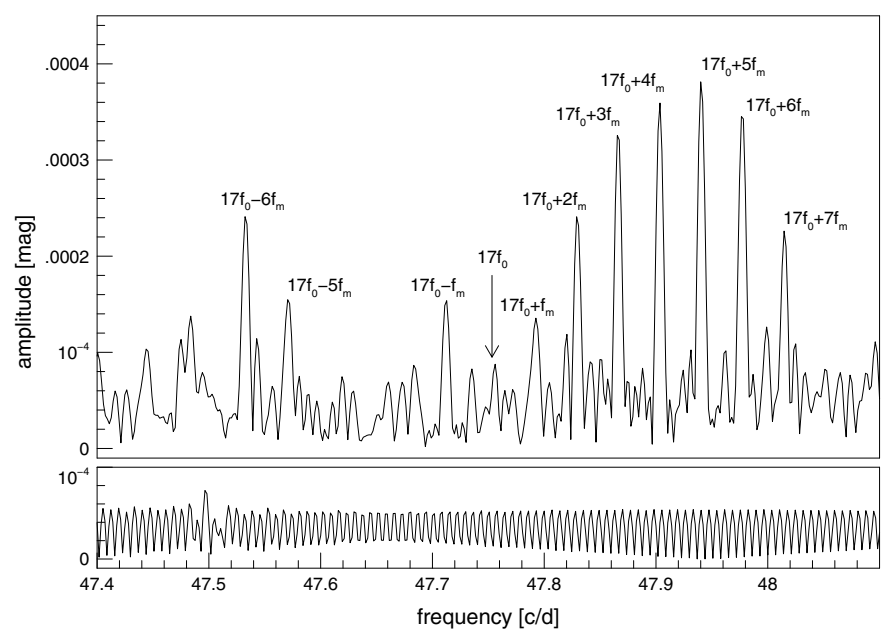

Fig. 5. Top: a part of the unbiased Fourier spectrum around the harmonics of $17 f_{0}$. Bottom: the same part of the window function in the same scale as the spectrum.

It is clear that more side peaks appear on the positive, higherfrequency sides $(k>0$, right) than on the negative, lowerfrequency sides (left). The asymmetry is caused by the amplitude difference between right and left side peaks. The right-side peaks have always a higher amplitude than the corresponding left-side ones.

To see the regularity in a particular case Fig. 5 shows the part of the Fourier spectrum around the $17 f_{0}$ harmonic. (The original

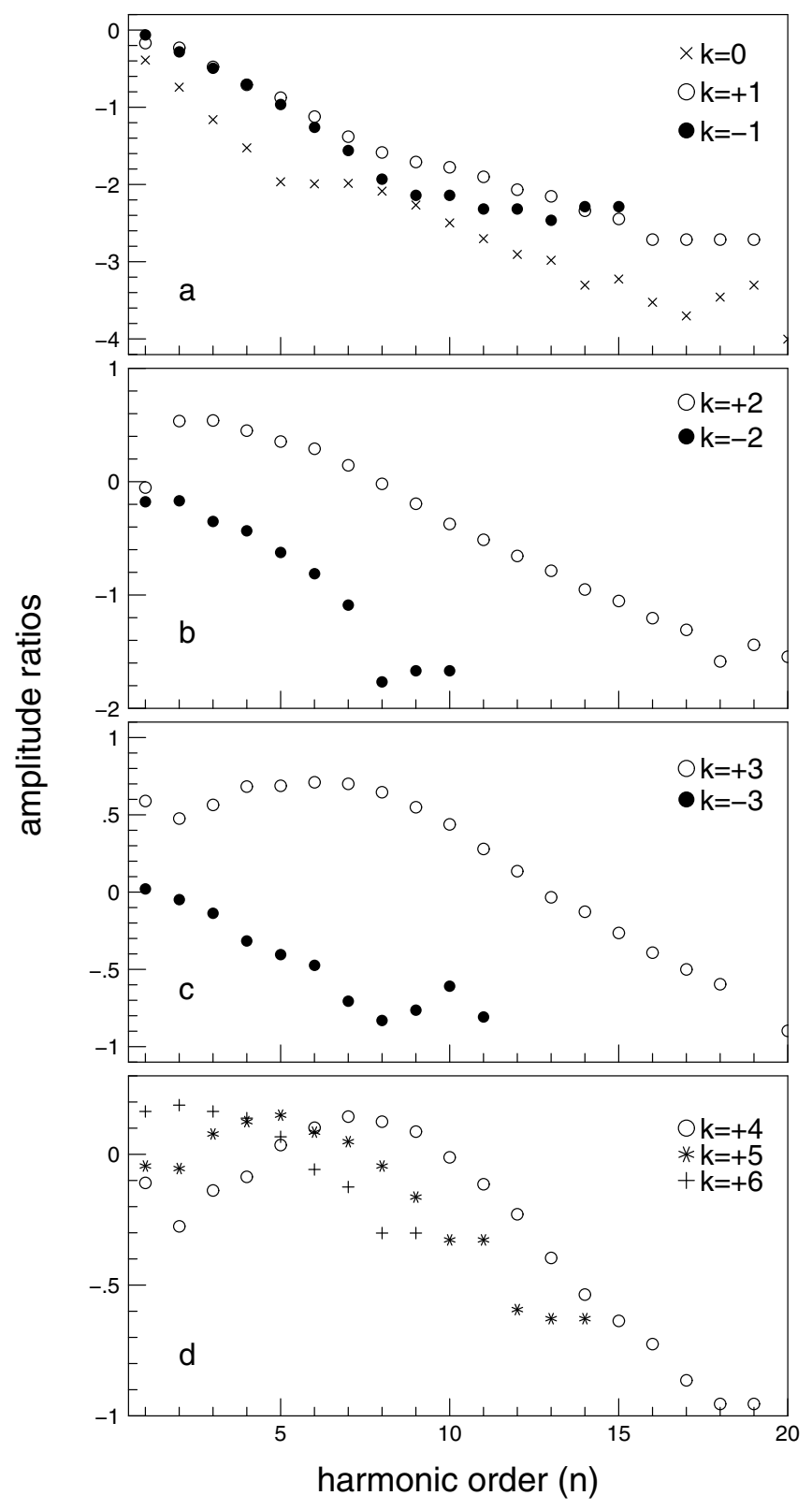

Fig. 6. a) Amplitude ratios of the harmonic components of the radial pulsation given in decimal logarithmic scale $\left(A_{n f_{0}} / A_{f_{0}}\right)$ compared to the amplitude ratios of the modulation components $\left(A_{\left.n f_{0}+f_{\mathrm{m}}\right)} / A_{f_{0}+f_{\mathrm{m}}}\right)$ and $\left(A_{\left.n f_{0}-f_{\mathrm{m}}\right)} / A_{f_{0}-f_{\mathrm{m}}}\right)$. b) Amplitude ratios of the modulation components $\left(A_{\left.n f_{0}+2 f_{\mathrm{m}}\right)} / A_{f_{0}+2 f_{\mathrm{m}}}\right)$ and $\left(A_{\left.n f_{0}-2 f_{\mathrm{m}}\right)} / A_{f_{0}-2 f_{\mathrm{m}}}\right)$. c) Amplitude ratios of the modulation components $\left(A_{\left.n f_{0}+3 f_{\mathrm{m}}\right)} / A_{f_{0}+3 f_{\mathrm{m}}}\right)$ and $\left.\left(A_{n f_{0}-3 f_{\mathrm{m}}}\right) / A_{f_{0}-3 f_{\mathrm{m}}}\right)$ and d) amplitude ratios of the modulation components $\left(A_{\left.n f_{0}+4 f_{\mathrm{m}}\right)} / A_{f_{0}+4 f_{\mathrm{m}}}\right)$, $\left(A_{\left.n f_{0}+5 f_{\mathrm{m}}\right)} / A_{f_{0}+5 f_{\mathrm{m}}}\right)$ and $\left(A_{\left.n f_{0}+6 f_{\mathrm{m}}\right)} / A_{f_{0}+6 f_{\mathrm{m}}}\right)$.

unbiased spectrum is plotted without prewhitening.) Although the harmonic at $17 f_{0}$ is not significant, its modulation side peaks are visible up to the 7 th order. The asymmetry between positive and negative sides is clearly detected. The maximal amplitude of the side peaks is reached at the 5th side peak (undecaplet). This regularity is similar for the side peaks around the main pulsation mode and its harmonics.

The general amplitude behaviour of the side peaks is presented in Fig. 6 for different kinds of multiplet peaks. The amplitude of triplet side peaks (left and right) runs parallel along straight lines on the logarithmic scale. There is a slight difference 
in the amplitude of side peaks between the 9th and 14th harmonics (panel a). There is a remarkable difference in the amplitude of the left and right-side peaks in the quintuplet structure. The right-side amplitudes (open circles) are much higher than the left-side peaks, although the inclination of the lines are similar. Only the right-side peaks around the main frequency and the first harmonics have as small amplitude as the left side peaks, much lower than around the other harmonics (panel b). There is also a large difference in the left and right side peaks in the case of the septuplet structure. The decrease of the amplitude is not parallel anymore, but there are larger differences around medium-order harmonics (panel c). The shape of the amplitude ratio vs. the harmonic order relation is similar for higher-order side peaks and for the lower-orders ones (panel d).

To characterize the asymmetry of the side peaks a parameter $\left.Q=\left(A_{+}-A_{-}\right) / A_{+}+A_{-}\right)$was calculated. In Table 2 we present the $Q$ values for the side peaks. This indicates a higher than average asymmetry to the right in the case of a triplet, nonuplet, undecaplet and quindecaplet structure, whereas the quintuplet, septuplet and tredecaplet structure shows a lower than average asymmetry to the right (see also Fig. 6). We computed the degree of deviation from the equidistant spacing of the multiplet structure with an uncertainty of \pm 0.0069 . The values of the degree of deviation are given in Table 2 with the uncertainties for all multiplet structures except for the quindecaplet.

We confirmed the previously known results obtained from ground-based observations for triplets and quintuplets. Our highprecision space data revealed new regularities in the higher-order side peaks, especially for the highest amplitude of the mediumorder right-side peaks. A comparison with other RR Lyrae stars will reveal whether the difference from a purely exponential decrease is connected to the more general parameters of the star or if it is only accidental in V1127 Aql.

\subsection{Additional frequencies}

A dominant peak $f^{\prime}=4.0326 \mathrm{~d}^{-1}$ was found in the course of our frequency analysis. A detailed analysis reveals a complex structure in the frequency domain around the dominant peak and around $f^{\prime \prime}=2.0163 \mathrm{~d}^{-1}$ presented in Fig. 7. A list of additional frequencies is given in Tables 1.B.1, 1.B.2, 1.B.3, 1.B.4, and 1.B.5. The combination terms between $f^{\prime}$ and $f_{0}$ exclude the possibility that $f^{\prime}$ and $f^{\prime \prime}$ are actually related to a background contact system, measured with V1127 Aql in the CoRoT window.

As we see in the figure and the tables, the dominant modes in the two regions are numerically connected to each other, $f^{\prime}=2 f^{\prime \prime}$ or $f^{\prime \prime}=f^{\prime} / 2$ depending on what we use as a reference frequency. Linear combination terms were also found with $f_{0}$ (up to $6 f_{0}$ ) and $f^{\prime}$ or $f^{\prime \prime}$. Many triplet, quintuplet, septuplet components of $f^{\prime}$ and $f^{\prime \prime}$ were identified with the Blazhko modulation frequency $f_{\mathrm{m}}$. Multiplet structures of these additional frequencies and their linear combinations with the main period and its harmonics were also found.

We could infer the presence of a second modulation-like frequency $f_{\mathrm{m} 1}=0.159 \mathrm{~d}^{-1}$. This frequency corresponds to a period of $6.289 \mathrm{~d}$. We did not observe this frequency itself in the Fourier spectrum, but many peaks could be identified as multiplet structures around the additional mode $f^{\prime}$ and its linear combinations with the main pulsation frequency $f^{\prime} \pm f_{0}$ and $f^{\prime}+k f_{0}(1<k<5$, Table 1C). A triplet structure was clearly detected and we always observed the right side peak to have a higher amplitude than the left one. A quintuplet structure was detected only around the $f^{\prime}$, $f^{\prime}+f_{0}$ and $f^{\prime}+2 f_{0}$ frequencies and showed on the left side peak.

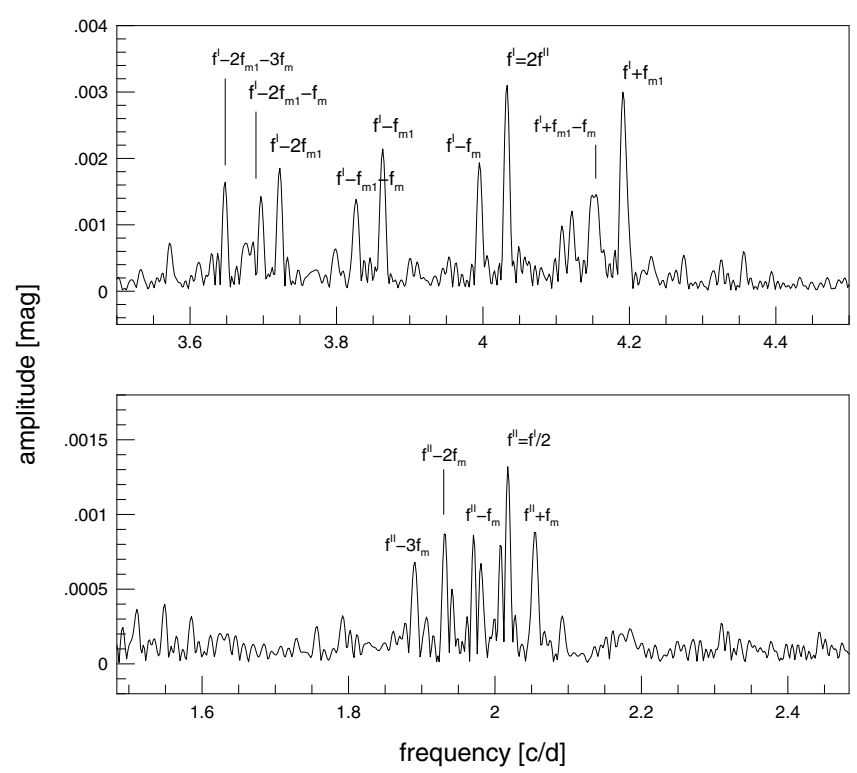

Fig. 7. Frequency pattern around the frequency of the additional mode and its harmonic $f^{\prime}=2 f^{\prime \prime}=4.0326 \mathrm{~d}^{-1}$ (top) and $f^{\prime \prime}=f^{\prime} / 2=$ $2.0163 \mathrm{~d}^{-1}$ (middle). Double peaks in the bottom panel are due to the fine structure of the window function. The panels are in the same frequency scale and are joined to each other.

A second modulation has been previously reported by Sódor et al. (2006); Sódor (2009), but it appeared around the main pulsation frequency, not around a rather low amplitude additional component. In our case second modulation peaks appear only in the interpretation of the additional modes and their linear combination of the main pulsation frequency.

It is a great challenge to find an answer to the origin of the additional frequency $f^{\prime}=4.0326 \mathrm{~d}^{-1}$, its subharmonic and its cyclic modulation $f_{\mathrm{m} 1}$. There are several possible explanations:

(1) the rotation period of V1127 Aql? But, the related period $1 / f^{\prime}=0.248 \mathrm{~d}$ seems to be too short to be the rotation period of the RRab star V1127 Aql. Peterson et al. (1996) measured the line-widths via cross-correlation for 27 field RR Lyrae stars and obtained an upper limit for the projected rotational velocity, which corresponds to $10-100 \mathrm{~d}$ of the rotation period;

(2) tidal effects in a binary system? If V1127 Aql has a companion, the $f^{\prime}=4.0326 \mathrm{~d}^{-1}$ would be the orbital period, but it seems to be too short to be connected to the orbital period. We could hardly explain the side peaks' structure around $f^{\prime}$ in this framework;

(3) Is V1127 Aql a double mode RR Lyrae variable? Additional frequencies with low amplitudes were also found in the photometric data obtained with the MOST satellite of AQ Leo, a "classical" RRd star (Gruberbauer et al. 2007). A higherorder radial or resonant non-radial mode was suggested by the authors to explain the two detected frequencies with a ratio of 2:1. In our case, V1127 Aql might be interpreted as oscillating in the fundamental radial mode $f_{0}=2.809017 \mathrm{~d}^{-1}$ $(0.355996 \mathrm{~d})$ and the first or a higher-order radial overtone mode $f^{\prime}=4.0326 \mathrm{~d}^{-1}(0.247978 \mathrm{~d})$. As in AQ Leo, the coupling terms are clearly detected as well. Moreover, both modes show cyclic modulation with $f_{\mathrm{m}}=0.0372 \mathrm{~d}^{-1}$. However, the ratio $f_{0} / f^{\prime}=0.6965$ and $f_{0}=2.809017 \mathrm{~d}^{-1}$ $(0.355996 \mathrm{~d})$ does not fit to the relation of the RR $d$ stars of our Galaxy. 


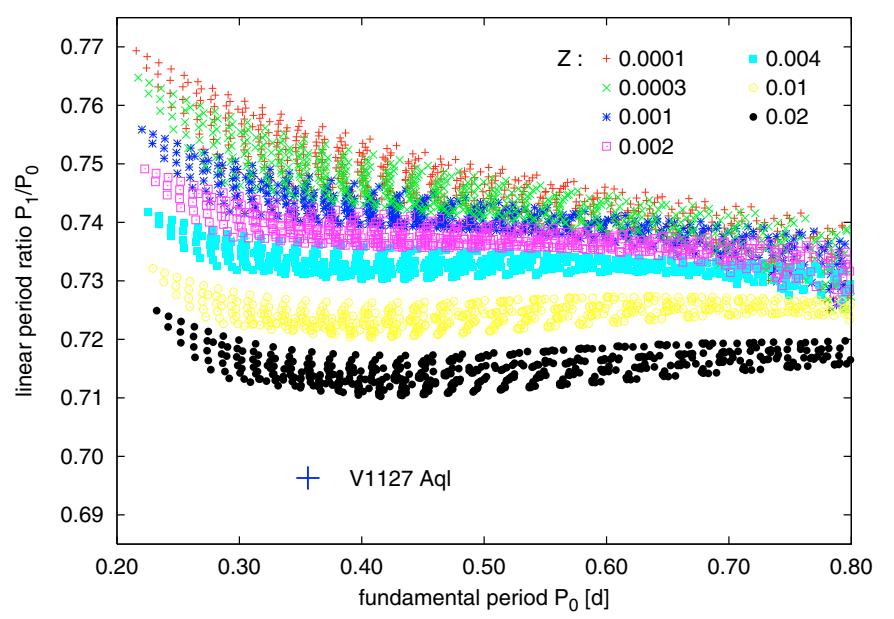

Fig. 8. Petersen diagram based on linear convective RR Lyrae models. The symbols denote different metallicities. The "+" sign shows the position of V1127 Aql assuming that the frequency $f^{\prime}$ is the first overtone mode.

To demonstrate this, we computed convective linear RR Lyrae model grids on an extremely large parameter space $\left(L=40,50,60\right.$ and $70 L_{\odot}, M=0.50-0.80 M_{\odot}$ with $\Delta M=0.05 M_{\odot}, T_{\text {eff }}=5000-8000 \mathrm{~K}, \Delta T_{\text {eff }}=100 \mathrm{~K}$, $Z=0.001,0.003,0.01,0.02$ and 0.04$)$. The other adopted parameters were standard RR Lyrae parameters (see Szabó et al. 2004). The Petersen diagram for different metallicities is shown in Fig. 8. The "+" sign marks the observed period ratio of $P^{\prime} / P_{0}=f_{0} / f^{\prime}$. We note that nonlinearity introduces a negligible difference in the periods and period ratios in this figure. Based on the large differences between the observed and computed period ratios we can exclude a radial-overtone explanation for the frequency $f^{\prime}$. It can be neither the first radial overtone nor one of the higher overtones, since the discrepancy between the computed higherovertone period ratios $\left(P_{i} / P_{0}\right)$ and $P^{\prime} / P_{0}$ is even larger than for the first overtone;

(4) is V1127 Aql a multiperiodic RR Lyrae variable? In this case, the frequency $f^{\prime}=4.0326 \mathrm{~d}^{-1}(0.248 \mathrm{~d})$ is a nonradial mode with a subharmonic $f^{\prime \prime}=f^{\prime} / 2=2.0163 \mathrm{~d}^{-1}$ and a cyclic modulation of $6.289 \mathrm{~d}$. In this scenario we have to suppose that the additional frequencies are non-radial modes. $f^{\prime}$ can be a non-radial p-mode while $f^{\prime \prime}$ could be interpreted as a g-mode, since it is longer than the radial fundamental mode. However, the numerical relation $\left(f^{\prime \prime}=f^{\prime} / 2\right)$ raised the possibility of $f^{\prime \prime}$ being a subharmonic. It would be interesting to check the possibility of a $2: 1$ resonance between a p-mode and a g-mode. For this purpose, however, non-radial non-linear codes for RR Lyrae stars would be required.

\subsection{Multimode pulsation}

We propose a second explanation that avoids the second modulation frequency. The $f_{\mathrm{m} 1}$ values are slightly different when considering the couples 4.0326, $4.1916\left(0.1590 \mathrm{~d}^{-1}\right)$ and 4.0326, $3.8632\left(0.1694 \mathrm{~d}^{-1}\right)$. Therefore, the $3.8632,4.0326$ and 4.1916 terms could be independent excited modes. Following this hypothesis, V1127 Aql could be a multimode pulsator, showing up to nine independent modes spanning the narrow interval from 3.64 to $4.82 \mathrm{~d}^{-1}$ (Table 1.C.1). Since the corresponding periods are shorter than the fundamental radial period, these terms can be typified as both radial modes belonging to a higher $n$ number (overtones) and non-radial modes. Looking at Fig. 8, we note that $f_{4}=3.722 \mathrm{~d}^{-1}$ and $f_{8}=3.749 \mathrm{~d}^{-1}$ supply period ratios compatible with the identification of one of them as the first overtone. Moreover, $f_{6}=4.825 \mathrm{~d}^{-1}$ supplies a period ratio compatible with the expected ratio between the second overtone and the fundamental mode. But, due to the large number, most if not all of these frequencies are likely related to non-radial modes. It it also noteworthy that the independent terms show a signature of an almost regular spacing (i.e., that between the 3.647, $3.674,3.697,3.722$, and $3.749 \mathrm{~d}^{-1}$ frequencies), which could be ascribed to effects (rotational splitting, large separation) typical of non-radial modes.

These additional terms show combinations with several harmonics of $f_{0}$ (up to $6 f_{0}$ ) with its Blazhko modulation (Table 1.C.2). We stress that the frequencies as listed in Table 1.C are the same listed in Table 1.B, but they are identified in a different way. There are some relations between the two solutions $\left(f_{\mathrm{m} 1}=f_{2}-f_{1},-f_{\mathrm{m} 1}=f_{3}-f_{1}, f^{\prime \prime}=f_{6}-f_{0}\right)$ and hence they could be considered to be very similar from a mathematical point of view.

It is very interesting to note that the terms $f_{1}, f_{2}$, and $f_{6}$ show some complete multiplet structures $f_{i} \pm k f_{\mathrm{m}}\left(k=2\right.$ for $f_{1}$ and $f-$ $2, k=3$ for $f_{6}$; Table 1.C.3). It is hard to say whether this directly means that the Blazhko phenomenon also affects other modes than the radial ones, or if these terms are just linear combinations since it could be, for instance, $f_{i}+f_{\mathrm{m}}=f_{i}+\left(f_{0}+2 f_{\mathrm{m}}\right)-\left(f_{0}+f_{\mathrm{m}}\right)$. In the former case these terms would have a relevant impact on the modelling of the underlying physical mechanism of the Blazhko effect, since $f_{\mathrm{m}}$ has been considered so far as a modulation of the fundamental radial mode $f_{0}$ only.

\section{The Blazhko phenomenon}

The most striking feature of V1127 Aql is the large amplitude and phase modulation of the light curve over five Blazhko cycles. We emphasize that five continuously covered Blazhko cycles have never been in anyone's hands for investigation. The present paper describes the regularity of the Blazhko modulation by traditional and new tools. Due to the proper sampling and high precision of the CoRoT data we were able to use distinguished points in the light curve to characterize the nature of the Blazhko modulation. Especially the use of the minimum of the light curve can be accounted as a new tool.

\subsection{Amplitude modulation}

Figure 9 shows the phased CoRoT light curve of V1127 Aql folded with the Blazhko period, $P_{\mathrm{m}}=26.88 \mathrm{~d}$. The modulation of V1127 Aql is highly non-linear, as can be judged from the non-sinusoidal modulation of the light curve in Fig. 9.

To assess the light variation characteristics of V1127 Aql, we plotted the maximum and minimum brightness as a function of time (i.e., the envelopes of the light curve), as shown in Fig. 10. Due to the non-linear nature of the modulation a thirdorder harmonic series of the Blazhko modulation frequency $\left(f_{\mathrm{m}}\right)$ is needed to separately fit the variations of the maxima and the minima, respectively. In this way, we obtain a total light variation of $0.753 \mathrm{mag}$. The maximum pulsation amplitude is $0.744 \mathrm{mag}$, while the minimum pulsation amplitude is 0.352 mag. Hence, the modulation amplitude amounts to $0.392 \mathrm{mag}$, where the magnitudes are in the CoRoT instrumental system. 


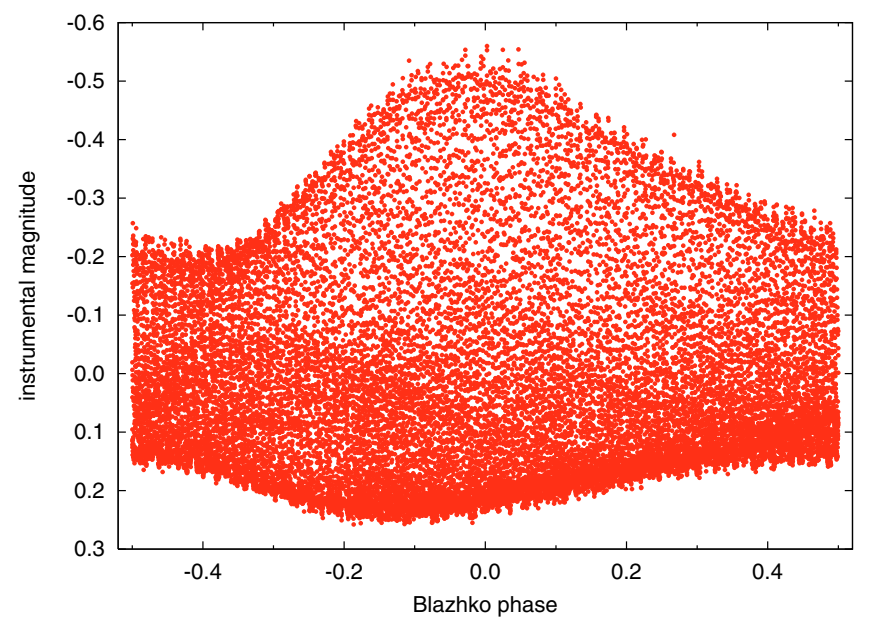

Fig. 9. The CoRoT light curve of V1127 Aql folded by the Blazhko period.

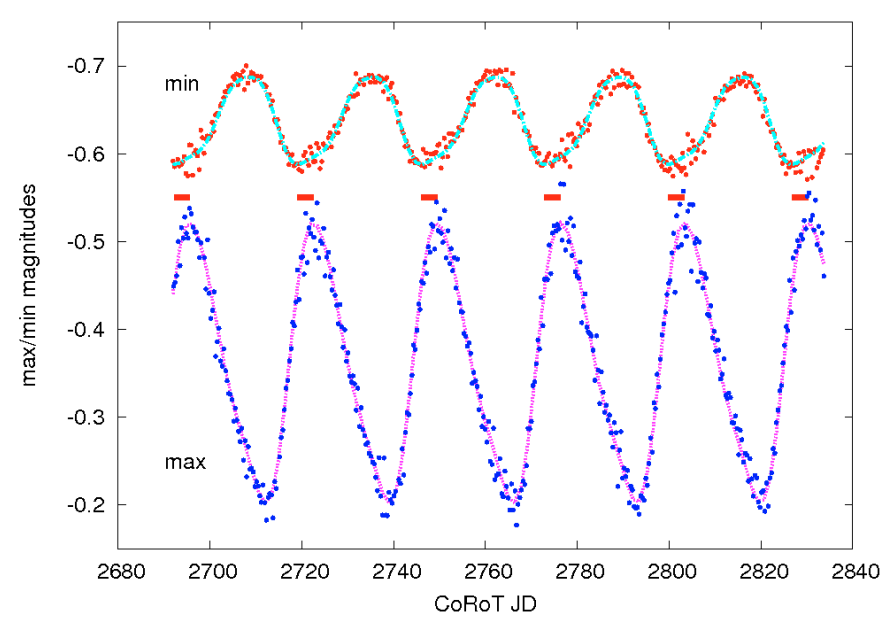

Fig. 10. Maximum and minimum (shifted by -0.9 vertically) magnitudes. Both curves were fitted with third order harmonic series. There is a phase lag between the maximum of maxima and the minimum of minima denoted by small line segments.

The light curve folded with the Blazkho period reveals a clear phase lag: the lowest minimum occurs around $\psi=-0.1$ and the highest maximum around $\psi=0.0$.

To visualize the phase lag between the maximum of maxima and the minimum of the minima, in Fig. 10 the minimum magnitudes were vertically shifted. From the fitted harmonic series we derive a shift of 3.4344 days which is equivalent to 0.1276 Blazhko phase.

\subsection{Phase modulation}

The $\mathrm{O}-\mathrm{C}$ curve is a traditional tool for the investigation of period changes. In the case of a Blazhko star we use it for the investigation of period variation seemingly caused by the phase modulation. We constructed $\mathrm{O}-\mathrm{C}$ curves of both the maxima and minima for the individual cycles (Fig. 11). Due to the higher scatter around the minimum of the light curve the minimum has never previously been used to construct an $\mathrm{O}-\mathrm{C}$ diagram. Note that because of its double minimum caused during the bump (Gillet \& Crowe 1988), the minimum is measured by fitting a polynomial

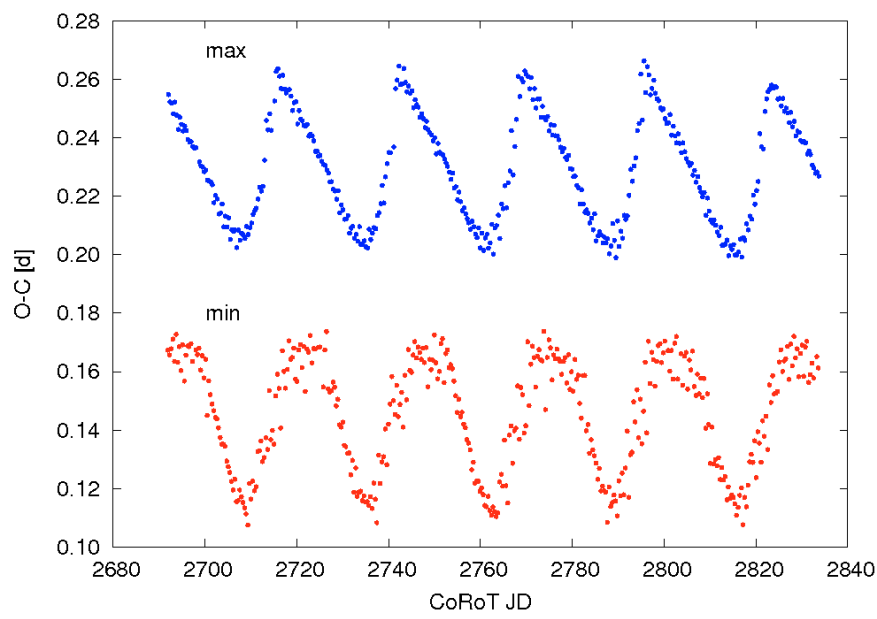

Fig. 11. The $\mathrm{O}-\mathrm{C}$ diagram for the pulsation maxima and minima.

of the 9th order. The same was found to give satisfactory results for the maxima, as well.

We used the epoch of the O-C values $T_{0}=2691.7543167$ CoRoTJD and the pulsation period $P_{0}=0.355996 \mathrm{~d}$. The variation of the maximum and minimum $\mathrm{O}-\mathrm{C}$ values is of the order of 0.06 days. The phase modulation reaches $14 \%$ of the pulsation period (around $77 \mathrm{~min}$ ). V1127 Aql exhibits one of the strongest phase modulations among all known Blazhko stars. Both the maximum and minimum $\mathrm{O}-\mathrm{C}$ diagrams reveal the same behaviour of the phase modulation.

\subsection{Instantaneous amplitude and period}

We interpret the phase modulation as a period variation. In order to find a connection between the amplitude modulation and the period variation we need to derive the time-dependent amplitude and period. As is well known, the $\mathrm{O}-\mathrm{C}$ diagram contains the integrated effect of the period variation. However, the numerical derivation of the $\mathrm{O}-\mathrm{C}$ data gives a rather noisy period value, which is an expected behaviour. One way to circumvent the problem is to fit the maximum (or minimum) $\mathrm{O}-\mathrm{C}$ values by a polynomial.

In this work we applied another approach, namely the analytical signal method (Kolláth et al. 2002). It uses the mathematical theorem that connects the real and imaginary part of any complex function which is analytic in the upper half plane. This relation makes it possible to unambiguously define the phase $\phi(t)$ and the amplitude $A(t)$ of a signal $a(t)=A(t) \mathrm{e}^{\mathrm{i} \phi(t)}$. Consequently it allows one to extract both the instantaneous amplitude and period.

Figure 12, shows the instantaneous period and the amplitude of the first Fourier-component of the dominant pulsational mode. Note that the longest period occurs near the smallest amplitude, and the shortest period is seen at the middle of the descending phase of the amplitude modulation. The average delay between the maximum period and maximum amplitude is $10.48 \pm 0.16$ days, corresponding to $0.390 \pm 0.006$ Blazhko phase.

The accuracy of the analytical signal method was checked through Monte-Carlo simulations by generating 1000 artificial datasets. Each dataset contained a modulated sine wave with similar amplitudes to the V1127 Aql light curve, using the same original light curve sampling. A Gaussian random error was added to the simulated data. The formal error of both quantities is less than the width of the lines in Fig. 12. 


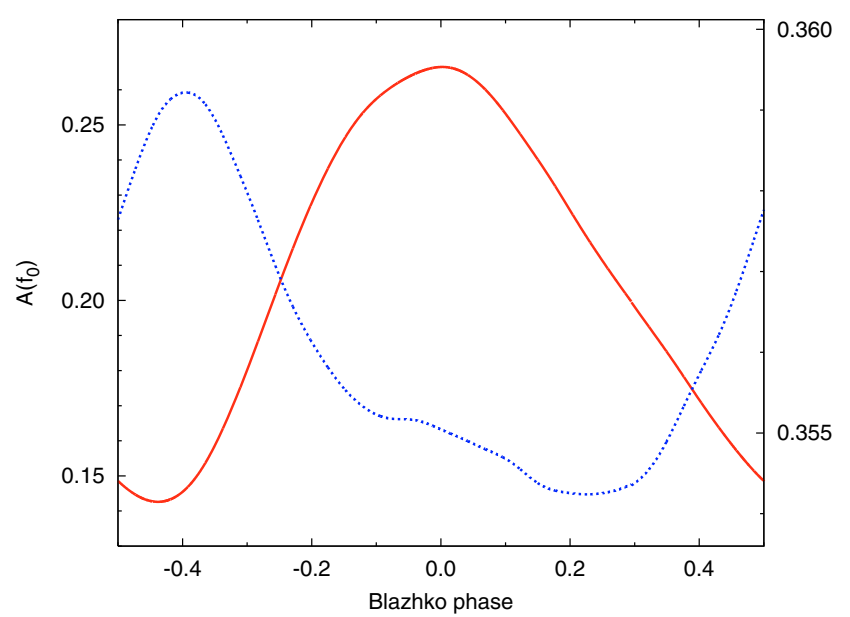

Fig. 12. The instantaneous period (dotted line) and the amplitude of the first Fourier-component of the dominant pulsational mode $A\left(f_{0}\right)$, (solid line) during the Blazhko modulation.

We applied the same analysis technique to three other Blazhko RR Lyrae stars found in the LRc01 field: 0101128793, 0100881648, 0101503544 (Szabó et al. 2009). The average phase delay between the amplitude and phase maxima is $0.534 \pm$ $0.053,0.469 \pm 0.012$ and $0.507 \pm 0.035$. Note that the four phaselag values are confined to a relatively small interval $[0.39 ; 0.53]$. Moreover, the phase lag in the case of MW Lyr is 0.38 , and 0.50 for DM Cyg (Jurcsik et al. 2008, 2009).

We argue that this quantity is an important parameter to study, because it is tightly connected to the physical mechanism of the amplitude and phase modulation. In addition, it strongly influences the multiplet structure of the modulated light curve (Benkő et al. 2009; Szeidl \& Jurcsik 2009).

The period variation emerges either due to a global parameter change in the star or because of non-linear effects or both, but its underlying cause and that of the amplitude modulation is not known. Therefore, the observed surprisingly small interval of the phase delay between the amplitude and the period variation presents a strong constraint for any model aiming to explain the Blazhko effect. The derivation of this quantity for more Blazhko stars is highly desirable.

From Fig. 12, it also seems that there is a continuous change in the pulsation period during the Blazhko cycle. The period change is antiphased with the light amplitude, showing an elbow centred at the phase of the Blazhko maximum. The full range of the period change is $0.006 \mathrm{~d}$ with $\delta P / P=+0.0169(1.69 \%$ of the pulsation period). The amplitude variation of the pulsation period is about $80 \%$ and the positive sign of $\delta P / P$ is consistent with the Stothers prediction (Stothers 2006).

\subsection{Maximum brightness and maximum phase variation}

The maximum brightness and maximum phase variation during the Blazhko phase are shown in Fig. 13. The maximum phase curve shows an asymmetric shape with a rapid increase on the rising branch (Blazhko phase between 0.4 and 0.7 ). The maximum phase plot was fitted using a 5th order Fourier-sum, and we fitted the maximum brightness curve using a 3th order Fouriersum. Table 3 lists the Fourier components of the maximum brightness and maximum phase fits. These differences show that the non-linearity is stronger in the phase modulation than in the amplitude modulation. The $0.0175 \mathrm{mag}$ and 0.0090 phase rms
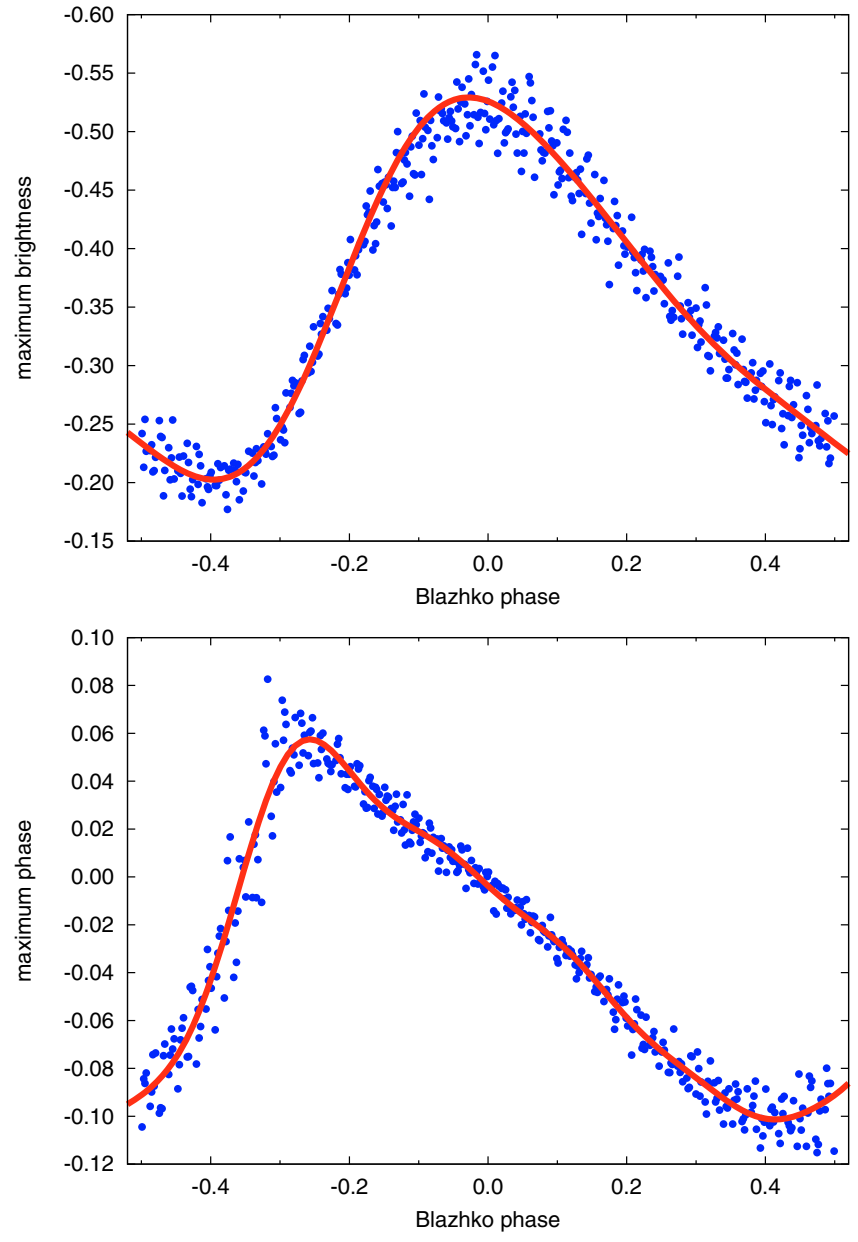

Fig. 13. a) Maximum brightness vs. Blazhko phase b) The same for the maximum phase.

scatter of the residuals of the maximum brightness and maximum phase fits are too large since the data are accurate within $\pm 0.005 \mathrm{mag}$ and $\pm 0.0018 \mathrm{~d}$, respectively. This means that both the maximum brightness and maximum phase curves reflect the imperfection of modelling the modulation using the Fourier sum of discrete frequency components.

The stability of the subsequent Blazhko cycles can be studied by plotting the maximum brightness values versus the maximum phase values. Figure 14 shows the curve for the first and the last observed Blazhko cycles, plotted with different symbols. The scatter is surprisingly large around the triangular shape curve and can neither be explained by the accuracy of the data nor by the procedure of determining the maximum phase and brightness values. We fitted the maximum brightness and the maximum phase values of each individual Blazhko cycle with a 5th order Fourier-sum, which allows us to discern a slight decrease in the maximum phase around 0.011 pulsation phase $(5.6 \mathrm{~min})$ during the five covered Blazhko cycles. This variation is quasi-linear, while there is a seemingly random variation in the maximum brightness values of the fits. A longer data set with the same accuracy would be necessary to decide whether the shift of the maximum phase continues or shows cyclic variations.

\section{Summary}

The photometric observations of V1127 Aql analysed in this paper, produced which were in the framework of CoRoT Exoplanet 
Table 3. Fourier amplitudes and phases of the fits to the maximum brightness and maximum phase data.

\begin{tabular}{ccccc}
\hline \hline Frequency & \multicolumn{2}{c}{ Max. brightness } & \multicolumn{2}{c}{ Max. phase } \\
\hline & ampl. & phase & ampl. & phase \\
\hline$f_{\mathrm{m}}$ & 0.154098 & 4.540907 & 0.069000 & 2.513789 \\
$2 f_{\mathrm{m}}$ & 0.033806 & 5.638383 & 0.020582 & 4.976442 \\
$3 f_{\mathrm{m}}$ & 0.006400 & 1.123083 & 0.009215 & 0.602859 \\
$4 f_{\mathrm{m}}$ & - & - & 0.004378 & 2.563744 \\
$5 f_{\mathrm{m}}$ & - & - & 0.002645 & 4.367358 \\
\hline
\end{tabular}

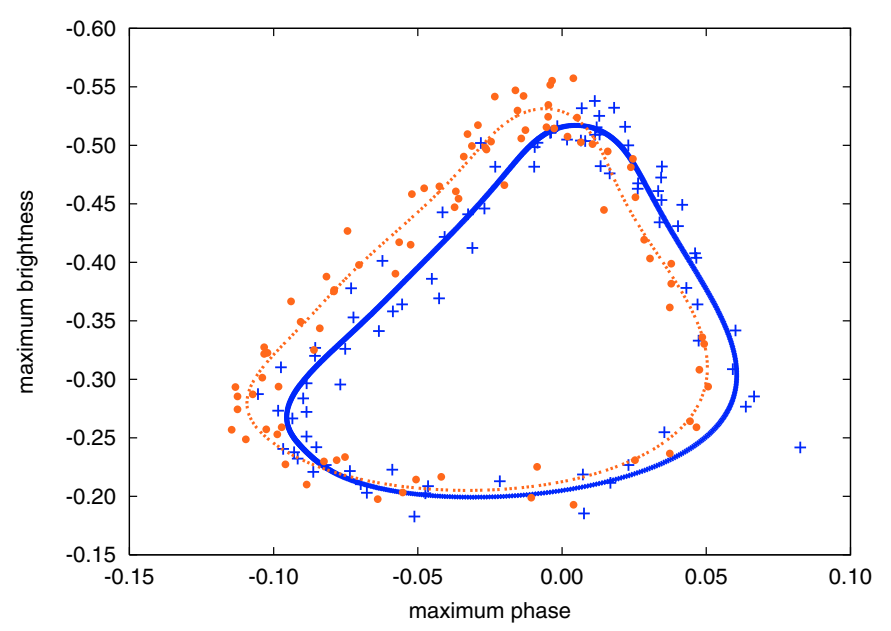

Fig. 14. a) Maximum brightness - maximum phase plot with 5th order harmonic fits. For clarity only the first (right, "+" symbols) and the fifth Blazhko cycles (left, dots) are shown.

programme, are the most accurate and continuous data set of a Blazhko star ever obtained.

Due to the proper data sampling and high precision we got new results on both the frequency content and the amplitude behaviour. We describe the still puzzling Blazhko phenomenon on a higher level, revealing more details to constrain the theoretical interpretation.

- We determined 18 harmonics of the main pulsation period. All of them have a complex structure of the Blazhko side peaks up to the septdecaplet (16 side peaks) distribution. Not all components of the side peaks' structure exceed the significance level.

- The $f_{\mathrm{m}}=0.0372 \mathrm{~d}^{-1}$ Blazhko modulation frequency as well as its first two harmonics were identified in the frequency spectrum. That is, the Blazhko modulation turned out to be non-linear both in amplitude and phase. We could determine that the phase modulation shows a higher non-linearity than the amplitude modulation.

- A frequency peak $f^{\prime}=4.0326 \mathrm{~d}^{-1}$ was found that does not fit the regular pattern of harmonics and Blazhko side peaks. Additionally, with a 2:1 frequency ratio $f^{\prime \prime}=2.0163 \mathrm{~d}^{-1}$ also appeared. Side peaks with the Blazhko modulation frequency $f_{\mathrm{m}}$ were also found around these peaks up to the quintuplet structure. We argue that rotation, tidal effects and higher-order radial overtones can be excluded as an explanation for the occurrence of those additional frequencies. A small possible amplitude modulation $f_{\mathrm{m} 1}$ was also detected. Since it is not connected to a radial mode, its interpretation is ambiguous. The most plausible interpretation is that nonradial p-modes are excited in V1127 Aql. We extended this explanation by removing $f^{\prime \prime}$ and $f_{\mathrm{m} 1}$ and providing a different identification of the detected frequencies in terms of a set of nine additional modes. These modes show linear combinations with the main pulsation period and the Blazhko modulation.

- Regarding the amplitude of the harmonics of the main frequency a special feature, a standstill, was observed at the medium-order harmonics. A similar feature was found in the monoperiodic non-Blazhko CoRoT star, ID:0101370131. Otherwise, the harmonics show an exponential decrease in amplitude. The distribution of Blazhko side peaks around the harmonics show a high-level asymmetry in favour of higher frequency side peaks because of the asymmetric amplitudes. The amplitude ratio of the lower- (left) and higher- (right) frequency side peaks have different values for the different components of the multiplet structure. The largest difference between the left and right components of the triplet side peaks is reached for medium-order harmonics (n). A similar behaviour is found for higher-order multiplets (k).

- As for the general characteristic of the Blazhko modulation, we derived the maximum value of the modulation of the light maximum (0.310) and at the minimum (0.100). Both values are robust compared to the 0.744 mag maximum amplitude brightness variation caused by the pulsation and are definitely not symmetrical to a constant mean brightness level. We derived a 3.4344 days (0.1276 Blazhko phase) phase lag between the maximum of maxima and minimum of minima. A strong 0.06 days modulation was derived, which amounts to $14 \%$ of the pulsation period. A long, 10.48 days (0.390 Blazhko phase) phase delay occured between the instantaneous period and amplitude during the Blazhko cycle.

- The phase delay between the amplitude and period modulation is 10.48 days (0.390 Blazhko phase) for V1127 Aql. This quantity is confined to a relatively small interval [0.38; 0.53 ] for all studied Blazkho stars which have a well covered light curve.

- The diagram of the maximum brightness and maximum phase over the five consecutive Blazhko cycles revealed a systematic shift. The shift between the first and the 5th cycle is 0.011 pulsational phase which means 5.6 min over 143 days.

Although we still cannot find a definite reason for the observed Blazhko modulation, our new results provide important constraints for the theoretical models to explain the phenomenon.

Acknowledgements. We would like to thank the referee for helpful comments. M.Ch. thanks the CoRoTeam and the CoRoT Variability Classifier (CVC Team) for their help during the preparation of the CoRoT data. J.M.B., M.P. and R.Sz. acknowledge the support of the ESA PECS projects No. 98022 \& 98114 . K.K. and E.G. thank the project FWF T359 and FWF P19962, and E.P. the Italian ESS project (contract ASI/INAF/I/015/07/0, WP 03170) for financial support.

\section{References}

Alcock, C., Allsman, R., Alves, D. R., et al. 2000, ApJ, 542, 257

Alcock, C., Alves, D. R., Becker, A., et al. 2003, ApJ, 598, 597

Auvergne, M., Bodin, P., Boisnard, L., et al. 2009, A\&A, 506, 411

Benkő, J. M., Paparó, M., Szabó, R., Chadid, M., et al. 2009, AIP Conf. Proc., 1170,273

Blazhko, S. 1907, Astr. Nachr., 175, 325

Bono, G., Caputo, F., Castellani, V., et al. 1997, ApJ, 483, 811

Borkowski, K. J. 1980, Acta Astron., 33, 393

Chadid, M., \& Chapellier, E. 2006, A\&A, 456, 305

Chadid, M., \& Gillet, D. 1997, A\&A, 319, 154

Chadid, M., Kolenberg, K., Aerts, C., \& Gillet, D. 1999, A\&A, 352, 201 
M. Chadid et al.: First CoRoT light curves of RR Lyrae stars

Chadid, M., Wade, G. A., Shorlin, S. L. S., \& Landstreet, J. D. 2004, A\&A, 413, 1087

Chadid, M., Vernin, J., \& Gillet, D. 2008, A\&A, 491, 537

Chadid, M., Baglin, A., Benkő, J. M., et al. 2009, AIP Conf. Proc., 1170, 235

Del Principe, M., Piersimoni, A. M., \& Bono, G. 2005, AJ, 129, 2714

Del Principe, M., Piersimoni, A. M., Storm, J., et al. 2006, ApJ, 652, 362

Dziembowski, W. A., \& Mizerski, T. 2004, Acta Astron., 54, 363

Gillet, D., \& Crowe, R. A. 1988, A\&A, 199, 242

Gessner, H. 1973, Veröff. Sternw. Sonneberg, 7, Heft 5, 525

Gruberbauer, M., Kolenberg, K., Rowe, J. F., et al. 2007, MNRAS, 379, 1498

Hoffmeister, C. 1966, AN, 289, 139

Hurta, Zs., Jurcsik, J., Szeidl, B., \& Sódor, Á. 2008, AJ, 135, 957

Jurcsik, J., Sódor, Á., Váradi, M., et al. 2005, A\&A, 430, 1049

Jurcsik, J., Szeidl, B., Sódor, Á., et al. 2006, AJ, 132, 61

Jurcsik, J., Sódor, Á., Hurta, Zs., et al. 2008, MNRAS, 391, 164

Jurcsik, J., Hurta, Zs., Sódor, Á., et al. 2009, MNRAS, 397, 350

Kolenberg, K., Smith, H. A., Gazeas, K. D., et al. 2006, A\&A, 459, 577

Kolenberg, K., Guggenberger, E., Medupe, T., et al. 2009, MNRAS, 396, 263

Kolláth, Z. 1990, Occ. Tech. Notes Konkoly Obs., Budapest, 1

Kolláth, Z., Buchler, J. R., Szabó, R., \& Csubry, Z. 2002, A\&A, 385, 932

Kovács, G. 1995, A\&A, 295, 693

Lenz, P., \& Breger, M. 2005, CoAst., 146, 53
Moskalik, P., \& Poretti, E., 2003, A\&A, 398, 213

Nagy, A. 1998, A\&A, 339, 440

Paparó, M., Szabó, R., Benkő, J. M., et al. 2009, AIP Conf. Proc., 1170, 240

Peterson, R. C., Carney, B. W., \& Latham, D. W. 1996, ApJ, 465, 47

Preston, G. W., Smak, J., \& Paczyński, B. 1965, ApJS, 12, 99

Reegen, P. 2007, A\&A, 467, 1353

Roberts, D. H., Lehar, J., \& Dreher, J. W. 1987, AJ, 93, 968

Sanna, N., Bono, G., Stetson, P. B., et al. 2009, ApJ, 699, L84

Shibahashi, H. 2000. ASP Conf. Ser., 203, 299

Smith, H. A., Church, J. A., Fournier, J., et al. 2003, PASP, 115, 43

Sódor, Á. 2009, CoAst, 159, 55

Sódor, Á., Vida, K., Jurcsik, J., et al. 2006, IBVS, 5705

Sollima, A., Cacciari, C., Arkharov, A. A. H., et al. 2008, MNRAS, 384, 1583

Stellingwerf, R. F. 1978, ApJ, 224, 953

Stothers, R. B. 2006, ApJ, 652, 643

Struve, O., \& Blaauw, A. 1949, ApJ, 108, 60

Szabó, R., Kolláth, Z., \& Buchler, J. R. 2004, A\&A, 425, 627

Szabó, R., Paparó, M., Benkő, J. M., Chadid, M., et al. 2009, AIP Conf. Proc., 1170,291

Szeidl, B., \& Jurcsik, J., 2009, CoAst, 160, 17

Walraven, Th. 1949, BAN, 11, 17 